\title{
Shape deformation of lipid membranes by banana-shaped protein rods: Comparison with isotropic inclusions and membrane rupture
}

\author{
Hiroshi Noguch: \\ Institute for Solid State Physics, University of Tokyo, Kashiwa, Chiba 277-8581, Japan
}

(Dated: September 3, 2018)

\begin{abstract}
The assembly of curved protein rods on fluid membranes is studied using implicit-solvent meshless membrane simulations. As the rod curvature increases, the rods on a membrane tube assemble along the azimuthal direction first and subsequently along the longitudinal direction. Here, we show that both transition curvatures decrease with increasing rod stiffness. For comparison, curvature-inducing isotropic inclusions are also simulated. When the isotropic inclusions have the same bending rigidity as the other membrane regions, the inclusions are uniformly distributed on the membrane tubes and vesicles even for large spontaneous curvature of the inclusions. However, the isotropic inclusions with much larger bending rigidity induce shape deformation and are concentrated on the region of a preferred curvature. For high rod density, high rod stiffness, and/or low line tension of the membrane edge, the rod assembly induces vesicle rupture, resulting in the formation of a high-genus vesicle. A gradual change in the curvature suppresses this rupture. Hence, large stress, compared to the edge tension, induced by the rod assembly is the key factor determining rupture. For rod curvature with the opposite sign to the vesicle curvature, membrane rupture induces inversion of the membrane, leading to division into multiple vesicles as well as formation of a high-genus vesicle.
\end{abstract}

PACS numbers: 87.16.D-,87.15.kt,87.16.A-

\section{INTRODUCTION}

In living cells, biomembranes often dynamically change their shapes to carry out their biological functions. Membrane budding, fusion, and fission occur during endo/exocytosis and vesicle transports. Cell organelles have specific shapes depending on their functions. Various types of proteins participate in regulation of these dynamic and static membrane shapes [1 6 ]. These proteins mainly control local membrane shapes in two ways: hydrophobic insertions (wedging) and scaffolding. In the former mechanism, a part of the protein, such as an amphipathic $\alpha$-helix, is inserted into the lipid bilayer membrane. In the latter mechanism, the protein domain has a strong affinity for the lipid polar head groups and adsorbs onto the lipid membrane. A BAR (Bin/Amphiphysin/Rvs) domain, which consists of a banana-shaped dimer, mainly bends the membrane along the domain axis via scaffolding [7-11]. Some of the BAR superfamily proteins, such as N-BAR proteins, also have hydrophobic insertions. Experimentally, the formation of membrane tubes and curvature-sensing by various types of BAR superfamily proteins have been observed 7 -21]. Dysfunctional BAR proteins are considered to be implicated in neurodegenerative, cardiovascular, and neoplastic diseases. Thus, it is important to understand the mechanism by which membrane shaping is regulated by proteins, not only from a basic science perspective but also for medical applications.

Homogeneous lipid membranes in a fluid phase are laterally isotropic and have zero spontaneous curvature. A

*noguchi@issp.u-tokyo.ac.jp local non-zero isotropic spontaneous curvature can be induced by adhesion of spherical colloids and polymeranchoring, as well as by transmembrane and other proteins 22]. Here, we call these objects that induce isotropic spontaneous curvature an isotropic inclusion. Their assembly into preferred curvature regions [23 27$]$ and membrane-mediated interactions between the colloids 28 30] have been previously explored.

In contrast, BAR domains, which are banana-shaped, generate an anisotropic curvature. Amphipathic $\alpha$ helices can also yield an anisotropic curvature 31]. Recently, the anisotropic nature of curvature has received increasing attention theoretically. The classical Canham-Helfrich curvature free energy 32, 33 has been extended to anisotropic curvatures $34-36]$. To simplify the interactions, the protein and membrane underneath it have been often modeled together as an undeformable object with a fixed curved shape such as a point-like object with an anisotropic curvature 37, 38] and a bent elliptical surface [39]. Furthermore, it has also been clarified that two undeformable parallel rods have an attractive interaction but the interaction is repulsive for a perpendicular orientation.

Atomic and coarse-grained molecular simulations 40 45] have been employed to investigate molecular-scale interactions between BAR proteins and lipids. The scaffold formation [43] and linear assembly [4] of BAR domains have been demonstrated. To investigate largescale membrane deformations, a dynamically triangulated membrane model [46, 47] and meshless membrane models [48 51] have been employed; consequently, various (meta)stable vesicle shapes [46 49] and the tubule formation dynamics [50] have been reported. Using meshless membrane and molecular simulations, vesicle rupture into high-genus vesicles has also been investi- 
gated [51, 52]. The high-genus vesicles obtained in this way resemble electron microscopic images of high-genus liposomes induced by N-BAR proteins well [51, 52]. Despite these numerous advancements, many questions related to the coupling between membrane shape deformation and the assembly of the protein rods remain.

In this paper, we focus on three questions: (i) How does protein elasticity modify protein assembly? (ii) How is rod assembly different from the isotropic inclusions? (iii) How is membrane rupture induced by protein rods? In the previous rupture simulations [51, 52], the effects of bending rigidity and rod curvature have been investigated but the other mechanical properties were not varied. We show here that the line tension of the membrane edge and the annealing speed, as well as the rod stiffness and density, are important parameters that determine the condition of membrane rupture.

In Sec. III the simulation model and method are described. We simulate membrane tubes and vesicles using an implicit-solvent meshless membrane model [4850, 53 [56]. A banana-shaped protein rod is assumed to be strongly adsorbed onto the membrane and the protein and membrane region below it are modeled as a linear string of particles with a bending stiffness and preferred curvature. In order to investigate the membranemediated interactions, no direct attractive interaction is considered between the rods.

In Sec. III the coupling between the assembly of the isotropic inclusions and shape deformation of membrane tubes and vesicles are presented. In Sec. IV] the assembly of the protein rods in the membrane tubes is shown. The dependence on the rod stiffness is investigated and the results are compared with those of the isotropic inclusions. In Sec. V] the vesicle rupture into high-genus vesicles and vesicle division are presented. The summary is given in Sec. VI]

\section{SIMULATION MODEL AND METHOD}

\section{A. Membrane Model}

We employ a spin meshless membrane model 48 50, [55]. The details of this meshless membrane model are described in Ref. 55. The position and orientational vectors of the $i$-th particle are $\mathbf{r}_{i}$ and $\mathbf{u}_{i}$, respectively. The membrane particles interact with each other via a potential,

$$
\begin{aligned}
\frac{U}{k_{\mathrm{B}} T}= & \sum_{i<j} U_{\mathrm{rep}}\left(r_{i, j}\right)+\varepsilon \sum_{i} U_{\mathrm{att}}\left(\rho_{i}\right) \\
& +\frac{k_{\mathrm{tilt}}}{2} \sum_{i<j}\left[\left(\mathbf{u}_{i} \cdot \hat{\mathbf{r}}_{i, j}\right)^{2}+\left(\mathbf{u}_{j} \cdot \hat{\mathbf{r}}_{i, j}\right)^{2}\right] w_{\mathrm{cv}}\left(r_{i, j}\right) \\
& +\frac{k_{\mathrm{bend}}}{2} \sum_{i<j}\left(\mathbf{u}_{i}-\mathbf{u}_{j}-C_{\mathrm{bd}} \hat{\mathbf{r}}_{i, j}\right)^{2} w_{\mathrm{cv}}\left(r_{i, j}\right),
\end{aligned}
$$

A laterally isotropic membrane inclusion is modeled as a membrane particle with $k_{\mathrm{r}}$ times larger bending rigidity and isotropic spontaneous curvature $C_{\text {iso }}$. For the neighbor pair of inclusions, $k_{\mathrm{tilt}}=k_{\mathrm{bend}}=k_{\mathrm{r}} k_{\mathrm{mb}}$ and $C_{\mathrm{bd}}=2 C_{\text {iso }} \sigma$ are employed in Eq. (11). For the pair of an inclusion and a membrane particle, averaged values are used as $k_{\mathrm{tilt}}=k_{\text {bend }}=k_{\mathrm{mb}}\left(k_{\mathrm{r}}+1\right) / 2$ and $C_{\mathrm{bd}}=C_{\mathrm{iso}} \sigma$.

where $\mathbf{r}_{i, j}=\mathbf{r}_{i}-\mathbf{r}_{j}, r_{i, j}=\left|\mathbf{r}_{i, j}\right|$, and $\hat{\mathbf{r}}_{i, j}=\mathbf{r}_{i, j} / r_{i, j}$. Each particle has an excluded volume with a diameter $\sigma$ that results from the repulsive potential, $U_{\text {rep }}(r)=$ $\exp [-20(r / \sigma-1)]$, with a cutoff at $r=2.4 \sigma$.

The second term in Eq. (1) represents the attractive interaction between particles. An attractive multibody potential $U_{\text {att }}\left(\rho_{i}\right)$ is employed to allow the formation of a fluid membrane over wide parameter ranges. The potential $U_{\text {att }}\left(\rho_{i}\right)$ is given by

$$
U_{\text {att }}\left(\rho_{i}\right)=0.25 \ln \left[1+\exp \left\{-4\left(\rho_{i}-\rho^{*}\right)\right\}\right]-C .
$$

Here, $\rho_{i}=\sum_{j \neq i} f_{\text {cut }}\left(r_{i, j}\right)$ and $C=0.25 \ln \left\{1+\exp \left(4 \rho^{*}\right)\right\}$, where $f_{\text {cut }}(r)$ is a $C^{\infty}$ cutoff function [54]

$$
f_{\text {cut }}(r)=\left\{\begin{array}{ll}
\exp \left\{A\left(1+\frac{1}{\left(r / r_{\text {cut }}\right)^{n}-1}\right)\right\} & \left(r<r_{\text {cut }}\right) \\
0 & \left(r \geq r_{\text {cut }}\right)
\end{array},\right.
$$

with $A=\ln (2)\left\{\left(r_{\text {cut }} / r_{\text {att }}\right)^{n}-1\right\}, r_{\text {att }}=1.9 \sigma\left(f_{\text {cut }}\left(r_{\text {att }}\right)=\right.$ $0.5)$, and the cutoff radius $r_{\text {cut }}=2.4 \sigma$. Here, $n=6$ is employed, as described in Ref. [48], instead of $n=12$, as described in Ref. [55], to use a less steep function. The density $\rho^{*}=7$ in $U_{\text {att }}\left(\rho_{i}\right)$ is the characteristic density at which the attraction is smoothly truncated. For $\rho_{i}<$ $\rho^{*}-1, U_{\text {att }}\left(\rho_{i}\right)$ acts as a pairwise attractive potential, while it approaches a constant value for $\rho_{i}>\rho^{*}+1$.

The third and fourth terms in Eq. (11) are discretized versions of the tilt and bending potentials, respectively. A smoothly truncated Gaussian function [54] is employed as the weight function

$$
w_{\mathrm{cv}}(r)=\left\{\begin{array}{ll}
\exp \left(\frac{\left(r / r_{\mathrm{ga}}\right)^{2}}{\left(r / r_{\mathrm{cc}}\right)^{n}-1}\right) & \left(r<r_{\mathrm{cc}}\right) \\
0 & \left(r \geq r_{\mathrm{cc}}\right)
\end{array},\right.
$$

where $n=4, r_{\mathrm{ga}}=1.5 \sigma$, and $r_{\mathrm{cc}}=3 \sigma$.

The membranes are in a fluid phase over a wide range of these parameters, and the properties of the fluid membrane can be widely varied. The spontaneous curvature $C_{0}$ of the membrane is given by $C_{0} \sigma=C_{\mathrm{bd}} / 2[55]$. For the membrane particles, which do not consist of proteins, $k_{\mathrm{tilt}}=k_{\mathrm{bend}}=k_{\mathrm{mb}}=10$ and $C_{\mathrm{bd}}=0$ are used. The bending rigidity $\kappa$ and edge tension $\Gamma$ can be controlled by $k_{\mathrm{mb}}$ and $\varepsilon$, respectively. $\kappa$ is a linear function of $k_{\mathrm{mb}}$ : $\kappa / k_{\mathrm{B}} T=1.77 k_{\mathrm{mb}}-2.5$ at $\varepsilon / k_{\mathrm{B}} T=5$, while $\kappa$ increases only $10 \%$ from $\varepsilon / k_{\mathrm{B}} T=3.5$ to 8 . $\Gamma$ is a monotonic increasing function of $\varepsilon: \Gamma \sigma / k_{\mathrm{B}} T=4.4,5.7$, and 6.8 at $\varepsilon / k_{\mathrm{B}} T=3.5,5$, and 8 , respectively. We fix $\varepsilon / k_{\mathrm{B}} T=5$ except for the vesicle-rupture simulations.

\section{B. Protein Model}


Note that, if the same values are used for a pair of an inclusion and a membrane particle, an additional attraction between the inclusions is induced by depletion, since the inclusion assembly reduces the area of the large bending rigidity. A similar attraction has previously been obtained for the binding sites of two membranes, when the membranes around the binding sites are hardened [56].

The protein rod is modeled as a linear chain of $N_{\mathrm{sg}}$ membrane particles. We use $N_{\mathrm{sg}}=10$ and a rod length of $r_{\text {rod }}=10 \sigma$, which corresponds to the typical aspect ratio of BAR domains. The BAR domain width is approximately $2 \mathrm{~nm}$, and its length ranges from 13 to $27 \mathrm{~nm}$ [8]. The protein particles in each protein rod are connected by a bond potential $U_{\text {rbond }} / k_{\mathrm{B}} T=$ $\left(k_{\text {rbond }} / 2 \sigma^{2}\right)\left(r_{i+1, i}-l_{\text {rod }}\right)^{2}$. The bending potential is given by $U_{\text {rbend }} / k_{\mathrm{B}} T=\left(k_{\text {rbend }} / 2\right)\left(\hat{\mathbf{r}}_{i+1, i} \cdot \hat{\mathbf{r}}_{i, i-1}-C_{\mathrm{r}}\right)^{2}$, where $C_{\mathrm{r}}=1-\left(C_{\text {rod }} l_{\text {rod }}\right)^{2} / 2$. We use $k_{\text {rbond }}=40$, $k_{\text {rbend }}=4000$, and $l_{\text {rod }}=1.15 \sigma$. The membrane potential parameters between neighboring protein particles in each rod are modified as $k_{\mathrm{tilt}}=k_{\mathrm{bend}}=k_{\mathrm{r}} k_{\mathrm{mb}}$ and $C_{\mathrm{bd}}=2 C_{\mathrm{rod}} \sigma$ in order to ensure bending of the rod along the normal to the membrane surface.

\section{Simulation Method}

The motion of the particle position $\mathbf{r}_{i}$ and the orientation $\mathbf{u}_{i}$ are given by underdamped Langevin equations:

$$
\begin{aligned}
\frac{d \mathbf{r}_{i}}{d t} & =\mathbf{v}_{i}, \quad \frac{d \mathbf{u}_{i}}{d t}=\boldsymbol{\omega}_{i}, \\
m \frac{d \mathbf{v}_{i}}{d t} & =-\zeta_{0} \mathbf{v}_{i}+\mathbf{g}_{i}^{0}(t)+\mathbf{f}_{i}, \\
I \frac{d \boldsymbol{\omega}_{i}}{d t} & =-\zeta_{\mathrm{r}} \boldsymbol{\omega}_{i}+\left(\mathbf{g}_{i}^{\mathrm{r}}(t)+\mathbf{f}_{i}^{\mathrm{r}}\right)^{\perp}+\lambda_{\mathrm{L}} \mathbf{u}_{i},
\end{aligned}
$$

where $m$ and $I$ are the mass and moment of inertia of the particle, respectively. The forces are given by $\mathbf{f}_{i}=-\partial U / \partial \mathbf{r}_{i}$ and $\mathbf{f}_{i}{ }^{\mathrm{r}}=-\partial U / \partial \mathbf{u}_{i}$ with the perpendicular component $\mathbf{a}^{\perp}=\mathbf{a}-\left(\mathbf{a} \cdot \mathbf{u}_{i}\right) \mathbf{u}_{i}$ and a Lagrange multiplier $\lambda_{\mathrm{L}}$ to keep $\mathbf{u}_{i}^{2}=1$. According to the fluctuationdissipation theorem, the friction coefficients $\zeta_{0}$ and $\zeta_{\mathrm{r}}$ and the Gaussian white noises $\mathbf{g}_{i}^{0}(t)$ and $\mathbf{g}_{i}^{\mathrm{r}}(t)$ obey the following relations of their averages and variances:

$$
\begin{aligned}
\left\langle g_{i, \alpha_{1}}^{\beta_{1}}(t)\right\rangle & =0, \\
\left\langle g_{i, \alpha_{1}}^{\beta_{1}}\left(t_{1}\right) g_{j, \alpha_{2}}^{\beta_{2}}\left(t_{2}\right)\right\rangle & =2 k_{\mathrm{B}} T \zeta_{\beta_{1}} \delta_{i j} \delta_{\alpha_{1} \alpha_{2}} \delta_{\beta_{1} \beta_{2}} \delta\left(t_{1}-t_{2}\right),
\end{aligned}
$$

where $\alpha_{1}, \alpha_{2} \in\{x, y, z\}$ and $\beta_{1}, \beta_{2} \in\{0, \mathrm{r}\}$. The Langevin equations are integrated by the leapfrog algorithm [57, 58]. In this study, we use $m=\zeta_{0} \tau_{0}, I=\zeta_{\mathrm{r}} \tau_{0}$, $\zeta_{\mathrm{r}}=\zeta_{0} \sigma^{2}$, and $\Delta t=0.005 \tau_{0}$ where $\tau_{0}=\zeta_{0} \sigma^{2} / k_{\mathrm{B}} T$. The simulation results are displayed with a time unit of $\tau=r_{\text {rod }}^{2} / D$, where $D$ is the diffusion coefficient of the membrane particles in the tensionless membranes; $D$ is calculated from the mean square displacement of the particles: $D \tau_{0} / r_{\text {rod }}^{2}=0.001 \pm 0.0001$, so that $\tau=1000 \tau_{0}$. This time unit is estimated as $\tau \sim 10^{-4} s$ from $r_{\text {rod }} \simeq 20$ $\mathrm{nm}$ and $D \simeq 4 \mu \mathrm{m}^{2} / \mathrm{s}$ of transmembrane proteins [59].
The assemblies of the isotropic inclusions and protein rods on membrane tubes and vesicles with $N=$ 2,400 were investigated at the inclusion density $\phi_{\text {iso }}=$ $N_{\text {iso }} / N=0.167$ and the $\operatorname{rod}$ density $\phi_{\text {rod }}=$ $N_{\text {rod }} N_{\mathrm{sg}} / N=0.167$, where $N_{\text {iso }}$ and $N_{\text {rod }}$ are the numbers of the isotropic inclusions and rods, respectively. The replica exchange molecular dynamics [60, 61] for $C_{\text {iso }}$ or $C_{\text {rod }}$ [48, 49] is used to obtain the thermal equilibrium states. Membrane rupture was investigated for vesicles with $N=9,600$ for $\phi_{\text {rod }} \geq 0.5$. The error bars are estimated from four and 10 independent runs for the replica exchange simulations and vesicle rupture, respectively.

The tube length is fixed in the longitudinal $(z)$ direction and periodic boundary conditions are employed. The radius of the tube is $R_{\text {cyl }}=0.989 r_{\text {rod }}$ for $L_{z}=$ $4.8 r_{\text {rod }}$. This tube radius is used, if not otherwise specified. In Sec. IV $L_{z}$ is also varied in order to investigate the tube radius dependence. The radii of the vesicles are $R_{\text {ves }}=1.54 r_{\text {rod }}$ and $3.07 r_{\text {rod }}$ at $N=2,400$ and 9,600, respectively, in the absence of the rods.

\section{ISOTROPIC INCLUSIONS}

First, we investigated the assembly of the isotropic inclusions in order to compare it with the rod assembly. When the bending rigidity difference $k_{\mathrm{r}}$ between the inclusions and membrane is small, the inclusions are isotropically distributed on a membrane tube and vesicle. However, the inclusion assembly occurs at $k_{\mathrm{r}} \gtrsim 6$ (see Figs. 11 and 2). The assembly regions have curvatures closer to the preferred curvature of the inclusions. Hence, for hard inclusions, assembly reduces the bending energy, which is greater than the loss of the mixing entropy.

\section{A. Membrane Tube}

For $C_{\text {iso }}=0$ at $k_{\mathrm{r}} \gtrsim 6$, the membrane tube deforms into an elliptic cylinder and the inclusions assemble into two longitudinal domains in two flatter regions [see Fig. 1(a)]. The tips of the ellipse have a large curvature, but the inclusions in the flatter regions can have a low bending energy; hence, the total bending energy is reduced. This phase separation in the azimuthal direction is captured by the amplitudes of the Fourier modes [see Figs. 1(e)-(h)]. The lowest Fourier modes of the membrane shape and inclusion density along the azimuthal $(\theta)$ direction are given by $r_{q \theta}=(1 / N) \sum_{i} r_{i} \exp \left(-2 \theta_{i} \mathrm{i}\right)$ and $n_{q \theta}=\left(1 / N_{\text {rod }}\right) \sum_{i} \exp \left(-2 \theta_{i} \mathrm{i}\right)$, respectively, where $\theta_{i}=\tan ^{-1}\left(x_{i} / y_{i}\right)$. In the axial $(z)$ direction, $r_{q z}=(1 / N) \sum_{i} r_{i} \exp \left(-2 \pi z_{i} \mathrm{i} / L_{z}\right)$ and $n_{q z}=$ $\left(1 / N_{\text {rod }}\right) \sum_{i} \exp \left(-2 \pi z_{i} \mathrm{i} / L_{z}\right)$. With increasing $k_{\mathrm{r}}$, the amplitudes of $r_{q \theta}$ and $n_{q \theta}$ along the $\theta$ direction increase concurrently at $C_{\text {iso }}=0$.

As $C_{\text {iso }}$ increases, the membrane also deforms sigmoidally along the axial $(z)$ direction and the inclusions 
(a) $\mathrm{C}_{\mathrm{iso}}=0$

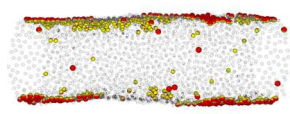

(b) $C_{\text {iso }} R_{\text {cyl }}=0.4$

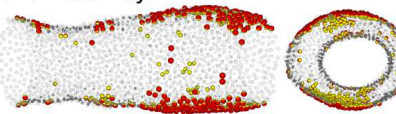

(c) $C_{\text {iso }} R_{\text {cyl }}=0.75$

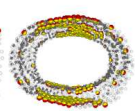

(d) $C_{i s o} R_{c y l}=1.5$

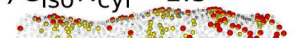

$\therefore \therefore 0^{\circ}$

$\therefore 6$.

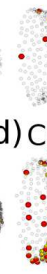

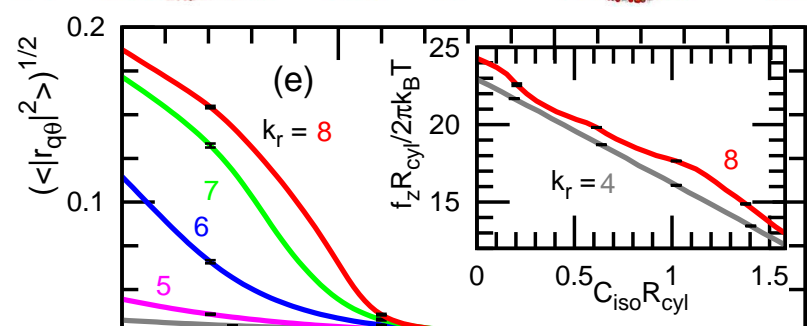
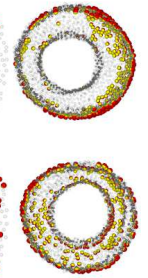

(a) $\mathrm{C}_{\text {iso }}=0$

(b) $C_{\text {iso }} R_{\text {ves }}=0.7$

(c) $\mathrm{C}_{\text {iso }} \mathrm{R}_{\text {ves }}=2$
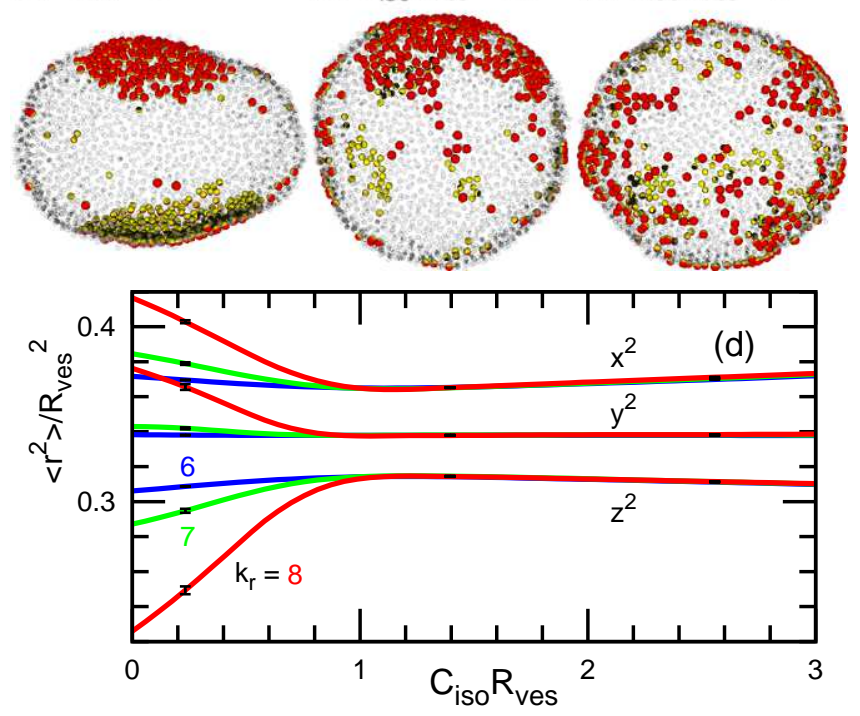

(f)

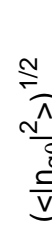

\section{0}
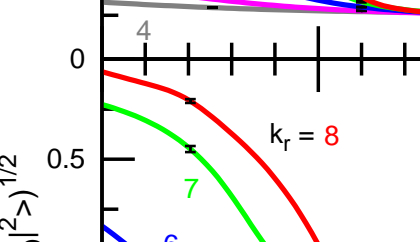

$++1++1+1$

$\frac{\mathrm{c}}{\mathrm{v}}$


FIG. 1: (Color online) Deformation of membrane tubes induced by isotropic inclusions at $\phi_{\text {iso }}=0.167$ and $N=2,400$. (a)-(d) Snapshots for (a) $C_{\text {iso }} R_{\text {cyl }}=0$, (b) 0.4 , (c) 1 , and (d) 1.5 at $k_{\mathrm{r}}=8$. The front and side views are shown. The inclusion is displayed as a sphere whose halves are colored in red (dark gray) and in yellow (light gray). The orientation vector $\mathbf{u}_{i}$ lies along the direction from the yellow (light gray) to red (dark gray) hemispheres. Transparent gray particles represent membrane particles. (e)-(h) Fourier amplitudes of (e), (f) membrane shape and (g), (h) the inclusion densities as functions of the spontaneous curvature $C_{\text {iso. The ampli- }}$ tudes of the lowest Fourier mode along the azimuthal $(\theta)$ and longitudinal $(z)$ directions are calculated for the membrane shape $\left(r_{q \theta}\right.$ and $\left.r_{q z}\right)$ and densities $\left(n_{q \theta}\right.$ and $\left.n_{q z}\right)$. The mean axial force $f_{z}$ of the membrane tube is shown in the inset of (e). Error bars are displayed at several data points.

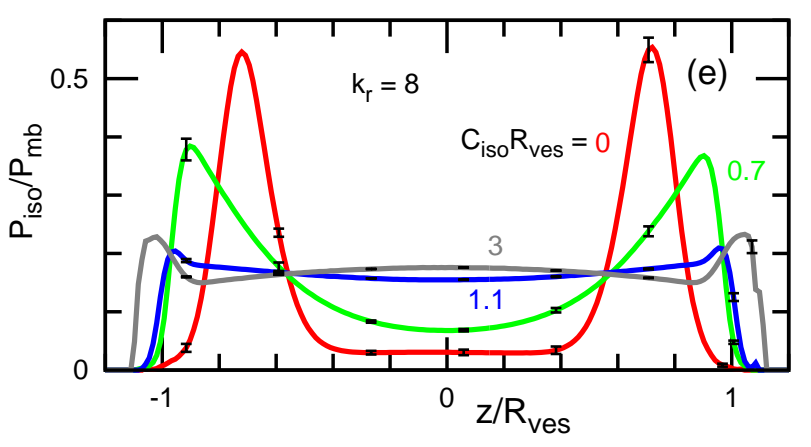

FIG. 2: (Color online) Vesicle deformation induced by isotropic inclusions at $\phi_{\text {iso }}=0.167$ and $N=2,400$. (a)-(c) Snapshots for (a) $C_{\text {iso }} R_{\text {ves }}=0$, (b) 0.7 , and (c) 2 at $k_{\mathrm{r}}=8$. (d) Three mean eigenvalues of the gyration tensor of the vesicle at $k_{\mathrm{r}}=6,7$, and 8 . (e) Probability distribution of the inclusions along the $z$ axis for $C_{\text {iso }} R_{\text {ves }}=0,0.7,1.1$, and 3 at $k_{\mathrm{r}}=8$. Error bars are displayed at several data points.

become concentrated in the convex region [see Fig. [1(b)]. As a result, the Fourier amplitudes of $\theta$ and $z$ decrease and increase, respectively. At $C_{\text {iso }} R_{\text {cyl }} \simeq 0.6$, the Fourier amplitudes of $\theta$ reach the values of the homogeneous membranes; the membrane deforms into a circular sigmoidal shape and the inclusions are concentrated along a convex ring [see Fig. 1(c)]. With a further increase in $C_{\text {iso }}$, the inclusions become uniformly distributed and the membrane is less undulated in the axial direction [see Figs. 1(d), (g), and (h)]. The spontaneous curvatures between the inclusion pair and between the membrane particle and inclusion are $C_{\text {iso }}$ and $C_{\text {iso }} / 2$, so that for $1 \lesssim C_{\text {iso }} R_{\text {cyl }} \lesssim 2$, local membrane spontaneous curvature can match the curvature $1 / R_{\text {cyl }}$ of the membrane tube.

An even further increase in $C_{\text {iso }}$ induces large membrane fluctuations, which induce the contact of the membranes at an hourglass-like neck of the membrane tube. This contact results in rupture of the membrane and for- 
mation of a spherical vesicle. At $k_{\mathrm{r}}=8$, the rupture occurs at $C_{\text {iso }} R_{\text {cyl }} \gtrsim 2$. With decreasing $k_{\mathrm{r}}$, larger values of $C_{\text {iso }}$ are required for the rupture.

In a cylindrical tube of a homogeneous membrane, the bending energy yields an axial force

$$
f_{z}=\left.\frac{\partial F}{\partial L_{z}}\right|_{A}=2 \pi \kappa\left(\frac{1}{R_{\mathrm{cyl}}}-C_{0}\right)
$$

since an increase in the axial length results in a decrease in the cylindrical radius, i.e., an increase in the membrane mean curvature [55]. At $k_{\mathrm{r}}=4$, the force $f_{z}$ linearly decreases with increasing $C_{\text {iso }}$ as shown in the inset of Fig. 1(e). This indicates that the inclusions are homogeneously mixed in the membrane. A similar linear dependence has previously been obtained for the density of anchored ideal-polymer chains 62]. In contrast, for $k_{\mathrm{r}}=8$, the $f_{z}-C_{\text {iso }}$ curve deviates from a straight line due to the inclusion assembly. A larger shape change induces greater deviation [compare the inset of Fig. 1 (e) with Figs. 1(g) and (h)].

\section{B. Vesicle}

Similarly to the membrane tube, at $C_{\text {iso }}=0$ and $k_{\mathrm{r}}=8$, the isotropic inclusions deform a vesicle into an oblate shape and the inclusions are concentrated in the two flatter regions [see Fig. 2(a)]. As $C_{\text {iso }}$ increases, the vesicle becomes more spherical and the inclusions are distributed more uniformly [see Figs. 2(b) and (c)]. For $C_{\text {iso }} R_{\text {ves }} \gtrsim 7$ at $k_{\mathrm{r}}=8$, vesicle division occurs and two spherical vesicles are formed.

These changes in the vesicle shape and inclusion assembly can be captured by the changes of the three principal lengths and inclusion distribution as shown in Figs. 2(d) and (e), respectively. The squared principal lengths $x^{2}, y^{2}$, and $z^{2}$ are the eigenvalues of the gyration tensor, $a_{\alpha \beta}=(1 / N) \sum_{j}\left(\alpha_{j}-\alpha_{\mathrm{G}}\right)\left(\beta_{j}-\beta_{\mathrm{G}}\right)$, where $\alpha, \beta \in x, y, z$ and $\alpha_{\mathrm{G}}$ is the center of mass. At $k_{\mathrm{r}}=6$, they are almost independent of $C_{\text {iso }}$ so that the vesicle maintains its spherical shape. Note that the differences among three eigenvalues at $k_{\mathrm{r}}=6$ are due to the thermal fluctuations. At $k_{\mathrm{r}}=7$, small deviations are recognized at $C_{\text {iso }} \simeq 0$ and at $k_{\mathrm{r}}=8$, a clear decrease in $\left\langle z^{2}\right\rangle$ is obtained. The inclusion distribution along the eigenvector of the smallest eigenvalue $(z)$ is calculated as $P_{\text {iso }} / P_{\mathrm{mb}}$, where $P_{\text {iso }}$ and $P_{\mathrm{mb}}$ are probabilities of finding the inclusions and all particles at each $z$ bin, respectively. The peaks of $P_{\mathrm{iso}} / P_{\mathrm{mb}}$ at both ends indicate the inclusion assembly on the flatter regions of the oblate vesicle [see the (red) solid line in Fig. 2(e)]. For $C_{\text {iso }} R_{\text {ves }} \gtrsim 1$, the inclusions are uniformly distributed.

Compared to the assembly of the anisotropic rods, as described in the next section, the inclusions assemble weakly and the shapes of the membrane tubes and vesicles fluctuate greatly, and for small values of $C_{\text {iso }}$, a single domain, instead of two domains, is occasionally formed.

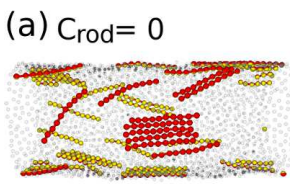

(b) $C_{\text {rod }} R_{\text {cyl }}=1$

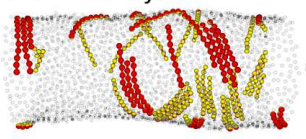

(c) $C_{\text {rod }} R_{\text {cyl }}=2$
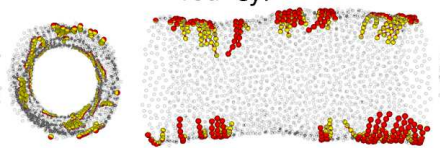

(d) $\mathrm{C}_{\text {rod }} R_{\text {cyl }}=3.5$

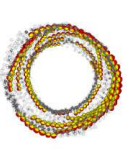

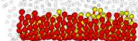

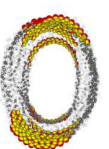

FIG. 3: (Color online) Snapshots of membrane tubes for (a) $C_{\text {rod }} R_{\text {cyl }}=0$, (b) 1 , (c) 2 , and (d) 3.5 at $k_{\mathrm{r}}=12$ and $\phi_{\text {rod }}=0.167$. The front and side views are shown. The protein rod is displayed as a chain of spheres whose halves are colored in red (dark gray) and in yellow (light gray).

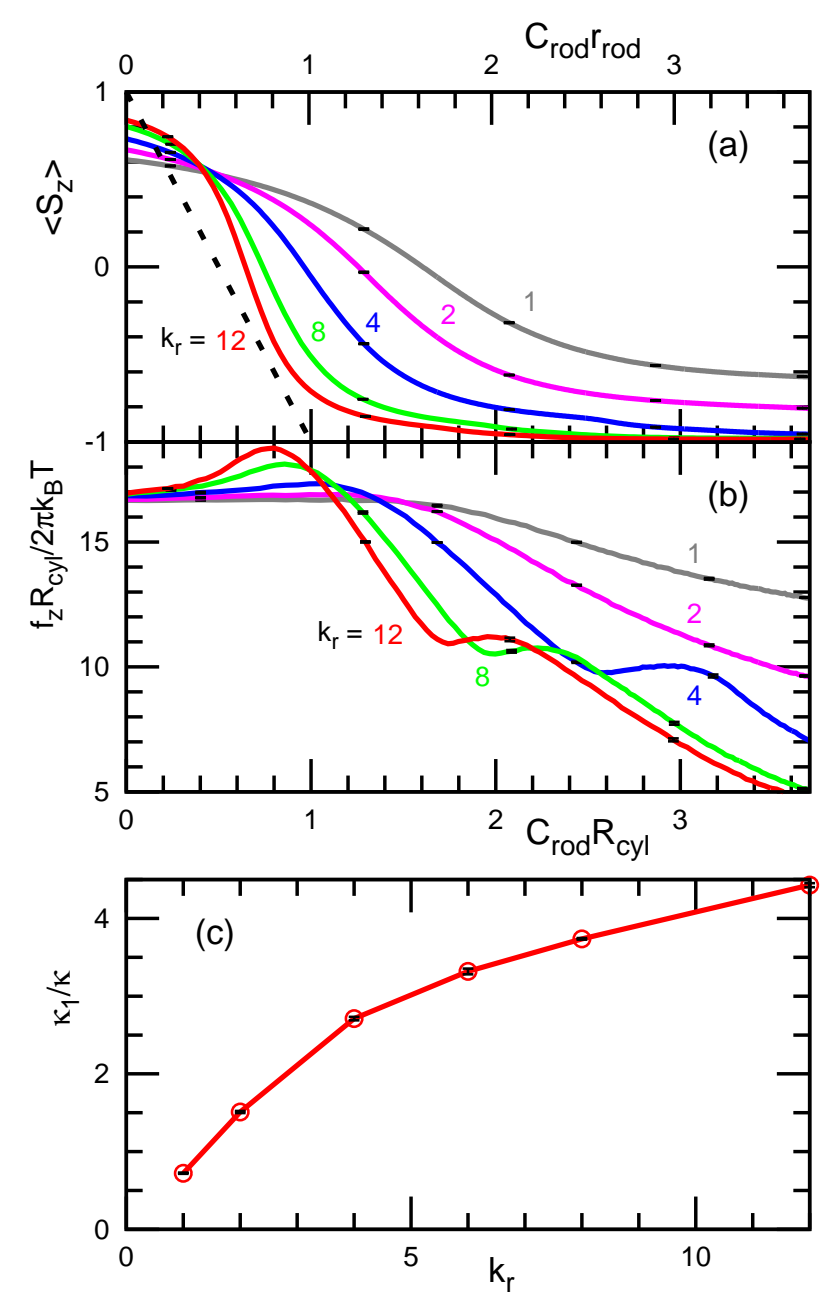

FIG. 4: (Color online) Rod orientation and axial force of the membrane tube at $\phi_{\text {rod }}=0.167$. (a), (b) Rod curvature, $C_{\text {rod }}$, dependence of (a) the orientation degree $S_{z}$ and (b) axial force $f_{z}$ at $k_{\mathrm{r}}=1,2,4,8$, and 12 . The (black) dashed line in (a) shows the relation of $S_{z}=1-2 C_{\mathrm{rod}} R_{\mathrm{cyl}}$ for undeformable rods without thermal fluctuations. (c) Effective bending rigidity of the rods estimated by Eq. (10). Error bars are displayed at several or all data points in (a) and (b) or (c), respectively. 


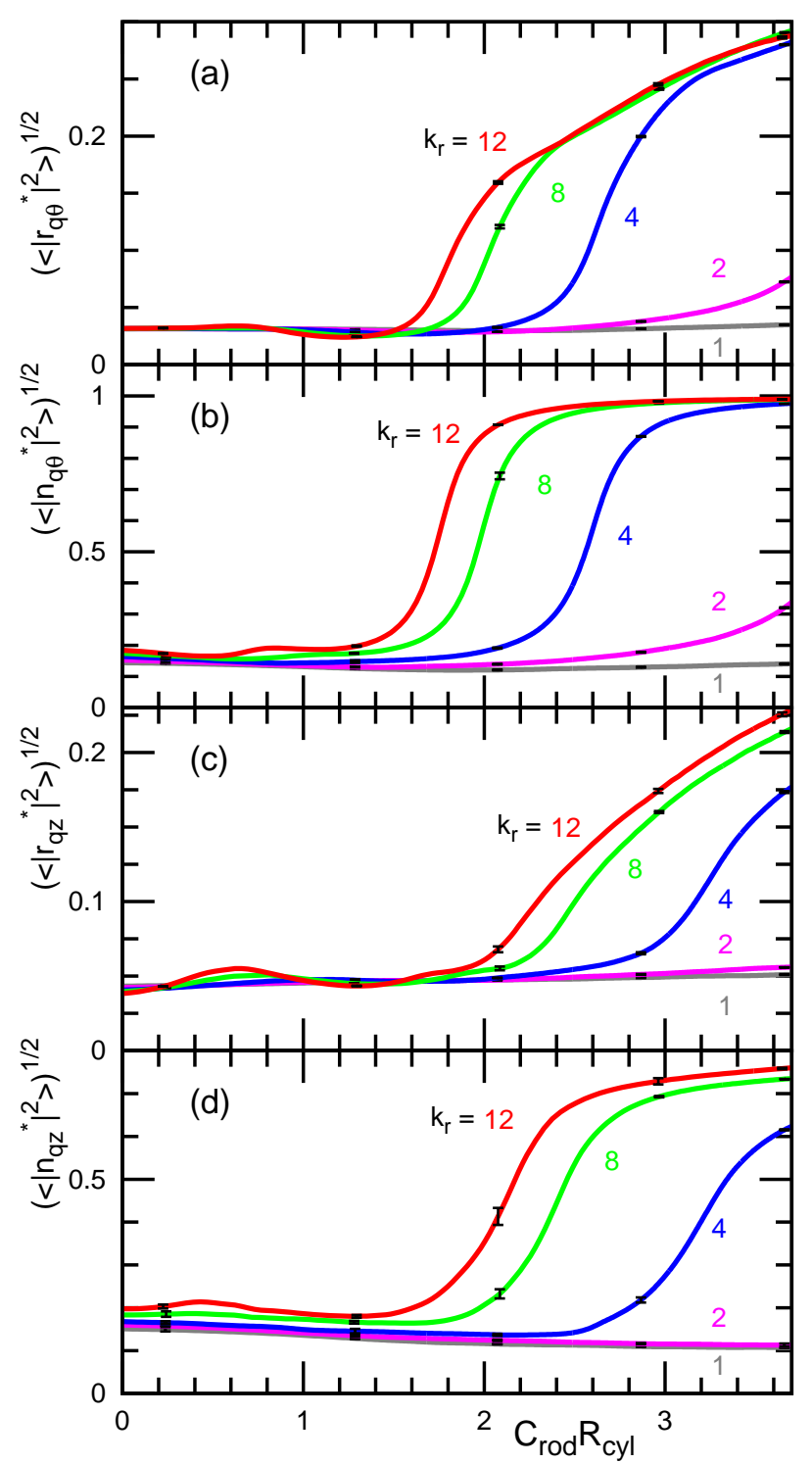

FIG. 5: (Color online) Rod curvature, $C_{\text {rod }}$, dependence of Fourier amplitudes of (a), (c) membrane shape and (b), (d) the rod densities at $\phi_{\text {rod }}=0.167$ and $k_{\mathrm{r}}=1,2,4,8$, and 12. The Fourier amplitudes are normalized by the values at $C_{\text {rod }}=0$ (denoted by the superscript $*$ ). Error bars are displayed at several data points.

High bending rigidity of the inclusion is required for assembly in the absence of direct attraction between the inclusions.

\section{MEMBRANE TUBE WITH PROTEIN RODS}

The protein rods exhibit a two-step assembly with increasing rod curvature $C_{\text {rod }}$ (see Figs. 36 6). In our previous papers [48, 49], we reported the assembly at $k_{\mathrm{r}}=4$. Here, we show the $k_{\mathrm{r}}$ dependence and summarize the assembly processes.
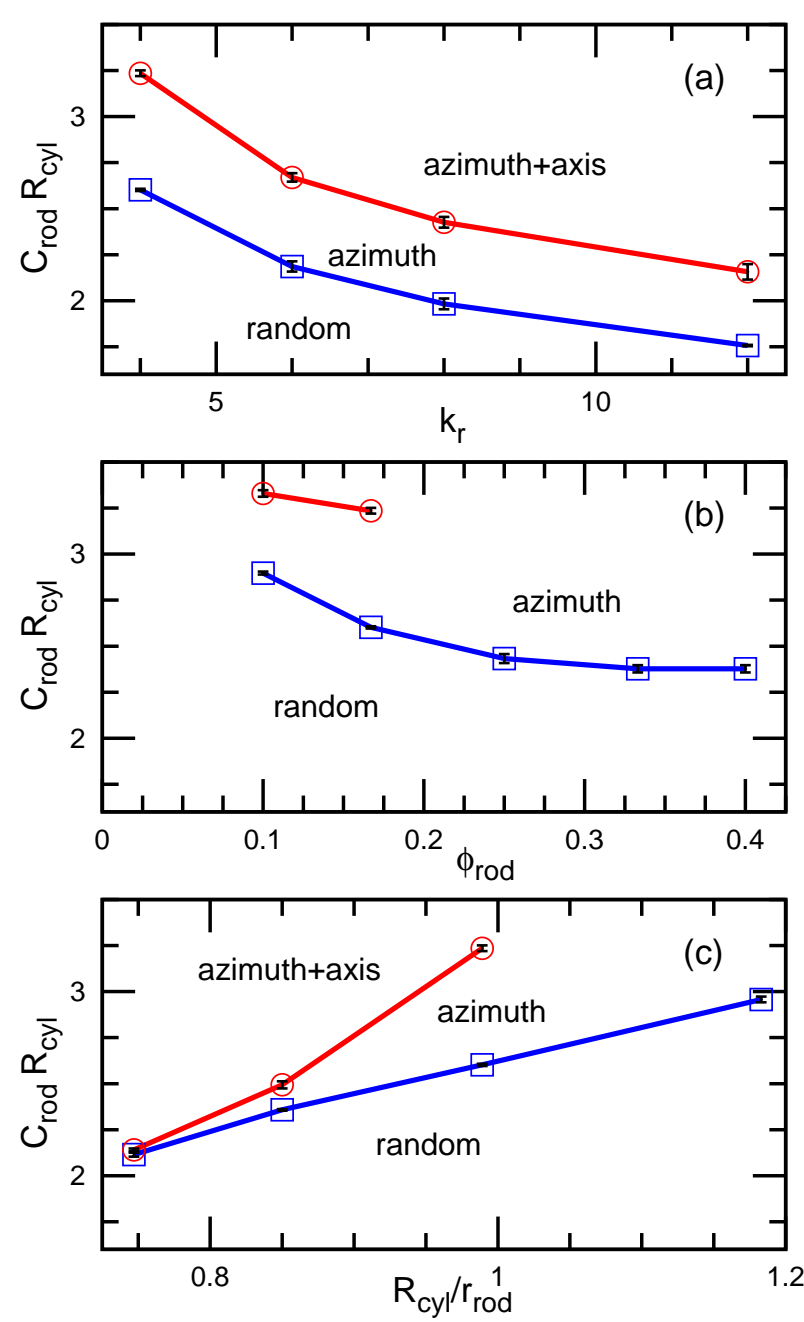

FIG. 6: (Color online) Phase diagrams of the membrane tube. (a) $C_{\text {rod }}-k_{\mathrm{r}}$ diagram at $\phi_{\text {rod }}=0.167$ and $R_{\text {cyl }}=0.989 r_{\text {rod. }}$. (b) $C_{\text {rod }}-\phi_{\text {rod }}$ diagram at $k_{\mathrm{r}}=4$ and $R_{\text {cyl }}=0.989 r_{\text {rod }}$. (c) $C_{\text {rod }}-R_{\text {cyl }} / r_{\text {rod }}$ diagram at $k_{\mathrm{r}}=4$ and $\phi_{\text {rod }}=0.167$.

At $C_{\text {rod }}=0$, the protein rods are oriented along the axial $(z)$ direction and uniformly distributed in the membrane tube [see Fig. B(a)]. As $C_{\text {rod }}$ increases, the rod orientation changes to the azimuthal $(\theta)$ direction and the mean orientational order parameter $\left\langle S_{z}\right\rangle$ decreases from 1 to -1 , where the orientational order parameter is defined as $S_{z}=\left(1 / N_{\text {rod }}\right) \sum_{i}\left(2 s_{i, z}{ }^{2}-1\right)$ [see Fig. 4(a)]. At larger $k_{\mathrm{r}},\left\langle S_{z}\right\rangle$ decreases more rapidly. For the limit of $k_{\mathrm{r}} \rightarrow \infty$, i.e., undeformable rods, the curvature along the rod axis is exactly $C_{\text {rod }}$. If the thermal fluctuations are neglected, a linear relation $S_{z}=1-2 C_{\text {rod }} R_{\text {cyl }}$ is obtained for $0 \leq C_{\text {rod }} R_{\text {cyl }} \leq 1$. As $k_{\mathrm{r}}$ increases, $\left\langle S_{z}\right\rangle$ approaches this linear relation, but relatively large deviations remain at $\left\langle S_{z}\right\rangle \simeq \pm 1$. These deviations are likely due to membrane undulations, which allow orientation fluctuations even for undeformable rods.

The axial force $f_{z}$ behaves differently from the closeto-linear dependence of the isotropic inclusions [compare 
Fig. 4(b) with the inset of Fig. 1(e)]. During orientation changes, $f_{z}$ is almost constant for small $k_{\mathrm{r}}$, while it increases slightly for large $k_{\mathrm{r}}$. This increase may be due to entropy reduction by tilted rods, since the tilted rods suppress membrane undulation in both the $z$ and $\theta$ directions. In this region $\left(0 \leq C_{\text {rod }} R_{\text {cyl }} \lesssim 1\right)$, changes in the Fourier amplitudes are very small in both directions for all values of $k_{\mathrm{r}}$ (see Fig. (5). Therefore, the membrane shapes and axial stress are modified only a little by the rods in this region.

With a further increase in $C_{\text {rod }}, f_{z}$ decreases linearly until the azimuthal assembly commences. When the rods are assumed to be completely oriented in the azimuthal direction, the axial force is given by [49],

$$
f_{z}=\frac{2 \pi \kappa}{R_{\mathrm{cyl}}}+2 \pi \phi_{\mathrm{rod}}\left(\frac{\kappa_{1}-\kappa}{R_{\mathrm{cyl}}}-\kappa_{1} C_{\mathrm{rod}}\right) .
$$

The first term is the force in the absence of rods. The second term is the force generated by the rods, which is proportional to $\phi_{\text {rod }}$ and linear with respect to $C_{\text {rod }}$. The effective bending rigidity $\kappa_{1}$ of the rods is estimated from the slope of $f_{z}-C_{\text {rod }}$ curves in the linear-decrease re-

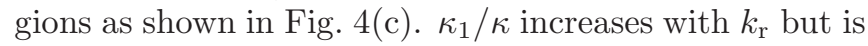
not linear with $k_{\mathrm{r}}$. This is because the orientation is not completely in the azimuthal direction and the interactions between rods and neighboring membrane particles are also involved in the bending deformation along the rod axis.

With an even further increase in $C_{\text {rod }}$, the rods assemble along the azimuthal direction and the membrane deforms into an elliptic tube [see Fig. 3(c)]. As $C_{\text {rod }}$ increases more, the rod assembly also occurs along the axial direction [see Fig. 3(d)]. The increases in the Fourier amplitudes of the azimuthal $(\theta)$ and axial $(z)$ modes indicate the azimuthal and axial assemblies, respectively (see Fig. 5). With increasing $k_{\mathrm{r}}$, both assemblies occur at smaller $C_{\text {rod }}$ [see Figs. 5 and[6(a)]. The curvatures $C_{\text {rod }}$ of the azimuthal and axial assembly points are determined by the inflection points of $\left(\left\langle\left|n_{q \theta}\right|^{2}\right\rangle\right)^{1 / 2}$ and $\left(\left\langle\left|n_{q z}\right|^{2}\right\rangle\right)^{1 / 2}$, respectively. The assembly is enhanced by the large rod stiffness in a manner similar to that of the isotropic inclusions. Thus, rod elasticity is one of the important factors that determine the assembly curvatures.

The phase diagram for the rod density $\phi_{\text {rod }}$ and the tube radius $R_{\text {cyl }}$ are shown in Figs. 6(b) and (c), respectively. For the azimuthal assembly, $\phi_{\text {rod }}$ gives an effect that is very similar to that of the rod stiffness $k_{\mathrm{r}}$. However, the axial assembly is different. At a large density $\left(\phi_{\text {rod }} \gtrsim 0.25\right)$, axial assembly does not occur, since the elliptic edges are filled by the rods [49]. As $\phi_{\text {rod }}$ increases further, the membrane deforms into a triangular or other polygonal tube, instead of the elliptic tube, and the rods assemble at the edges of the polygonal tube [49]. As $R_{\text {cyl }}$ increases, a slightly larger $C_{\text {rod }} R_{\text {cyl }}$ is needed for assembly in both directions. At a large tube radius $\left(R_{\text {cyl }} / r_{\text {rod }} \gtrsim 1\right)$, axial assembly does not occur for the same reason as for a large $\phi_{\text {rod }}$. The length $L_{z}$ decreases as $L_{z} \propto 1 / R_{\text {cyl }}$ for the constant membrane area, so that the elliptic edges can be filled by a smaller number of rods.

Protein rods with large rod curvatures form a tight assembly in the membrane tubes as well as in the vesicles [48, 49]. It differs from the assembly of isotropic inclusions into flat membranes, which requires a larger bending elasticity. The rod assembly forms a saddle shape in the membrane tube. In contrast, such a saddle membrane is not stabilized by isotropic inclusions with a large positive spontaneous curvature since its mean curvature is small.

\section{VESICLE RUPTURE}

Membranes can rupture under a large stress. It is determined by the competition between the membrane deforming force (by the protein rods in this study) and the line tension $\Gamma$ of the membrane edge. For example, under a large positive surface tension $\gamma$, a membrane pore can expand until the pore radius reaches the stable value $R_{\text {pore }}=\gamma / \Gamma[54,63]$. A vesicle can spontaneously transform into a disk-shaped flat membrane when $\Gamma<(2 \kappa+\bar{\kappa}) / R_{\text {ves }}[6466$. Here, we use sufficiently large $\Gamma$ to prevent membrane rupture in the absence of rods.

Figures 7] 9 show vesicle shapes resulting from membrane rupture by rod bending. Rupture changes vesicle topology. Figure 7 shows the vesicle shapes as the rod curvature changed suddenly from $C_{\text {rod }} R_{\text {ves }}=0$ to 6.2 . As the edge tension $\Gamma$ decreases, the membrane is ruptured more frequently and vesicles with higher genus $g$ are formed [see Figs. I (e) and (f)]. As the rod stiffness $k_{\mathrm{r}}$ or rod density $\phi_{\text {rod }}$ increases, the rods deform the membrane more rapidly and membrane rupture is enhanced. For $\langle g\rangle \lesssim 8,\langle g\rangle$ depends linearly on $\Gamma$ while it is saturated at $\langle g\rangle \simeq 10$. A greater number of $\langle g\rangle$ indicates the occurrence of more rupture. The obtained values of $g$ have a narrow distribution as indicated in small error bars that represent standard errors for 10 samples. The saturation of $\langle g\rangle$ is likely caused by the upper limit of genus, $g \simeq 13$, which is determined by the vesicle size. A higher genus can be obtained for larger vesicles. The obtained high-genus vesicles, shown in Fig. 77(a), agree with the previously reported shapes in simulations and experiments [51, 52]. Here, we have clarified that the edge tension and rod stiffness and density are important factors for vesicle rupture. At low $\phi_{\text {rod }}$, rod-free membranes are phase-separated and form flat regions while the rod assemblies form tubes and semicylindrical edges of the flat membranes [see Figs. 7(c) and (d)]. A similar coexistence of tubular networks and flat membranes is seen in endoplasmic reticulum (ER) [2]. When $C_{\text {rod }}$ changes slowly rather than rapidly, vesicle rupture is suppressed. The lower (red) line in Fig. 9(b) shows $\langle g\rangle$ as a function of the annealing time $t_{\mathrm{an}}$, for which $C_{\mathrm{rod}}(t)$ is changed linearly from $C_{\text {rod }} R_{\text {ves }}=0$ to 6.2 . No vesicles are ruptured at $t_{\mathrm{an}}=40 \tau(\sim 4 \mathrm{~ms})$. Thus, the rapid adsorption 

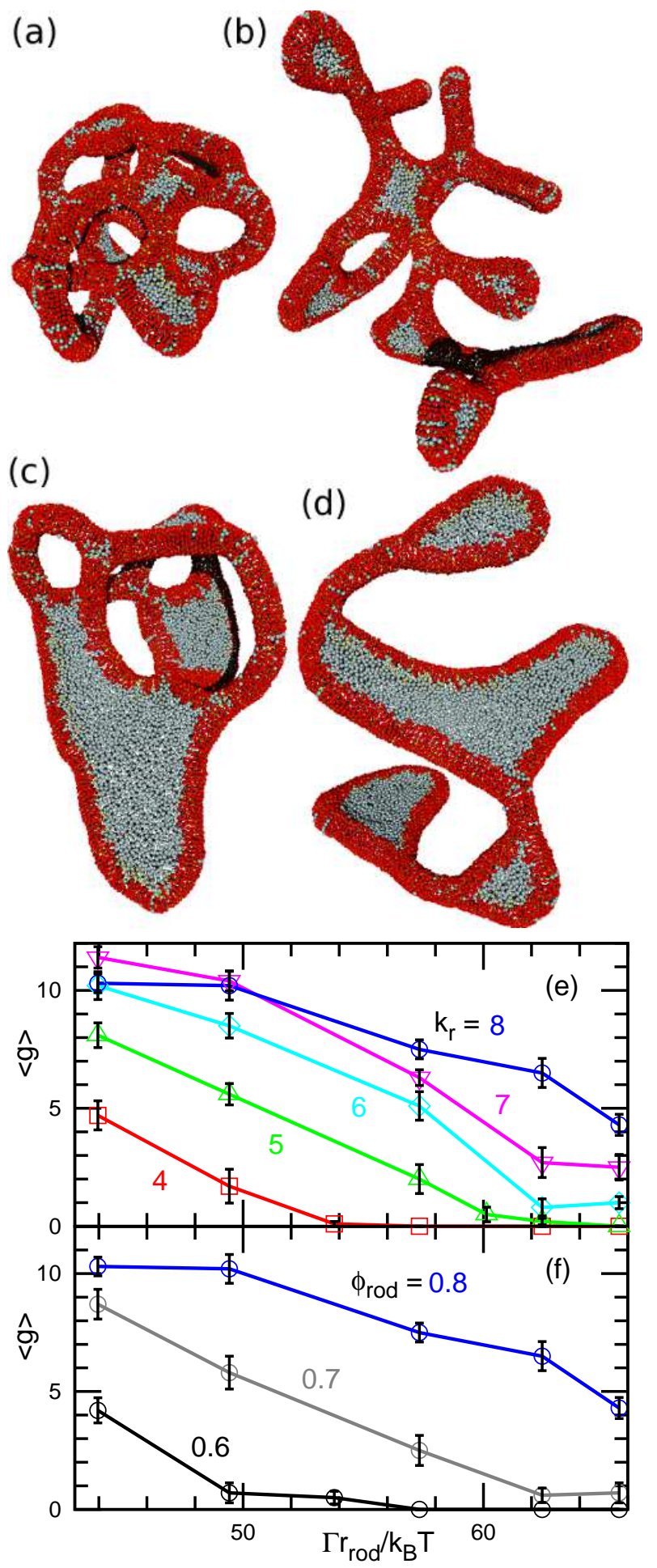

FIG. 7: (Color online) Membrane rupture at $C_{\text {rod }} R_{\text {ves }}=6.2$ and $N=9,600$. (a)-(d) Snapshots of vesicles. (a) Genus-11 vesicle at $\Gamma r_{\text {rod }} / k_{\mathrm{B}} T=49, k_{\mathrm{r}}=6$, and $\phi_{\text {rod }}=0.8$. (b) Genus1 vesicle at $\Gamma r_{\text {rod }} / k_{\mathrm{B}} T=66, k_{\mathrm{r}}=6$, and $\phi_{\text {rod }}=0.8$. (c) Genus- 5 vesicle at $\Gamma r_{\text {rod }} / k_{\mathrm{B}} T=44, k_{\mathrm{r}}=8$, and $\phi_{\text {rod }}=0.6$. (d) Genus- 0 vesicle at $\Gamma r_{\text {rod }} / k_{\mathrm{B}} T=57, k_{\mathrm{r}}=8$, and $\phi_{\text {rod }}=$ 0.6. Membrane particles are displayed by nontransparent gray particles. (e)-(f) Mean number of the genus $\langle g\rangle$ of vesicles as functions of the line tension $\Gamma$. (e) $k_{\mathrm{r}}=4,5,6,7$, and 8 for $\phi_{\text {rod }}=0.8$. (f) $\phi_{\text {rod }}=0.6,0.7$, and 0.8 for $k_{\mathrm{r}}=8$.

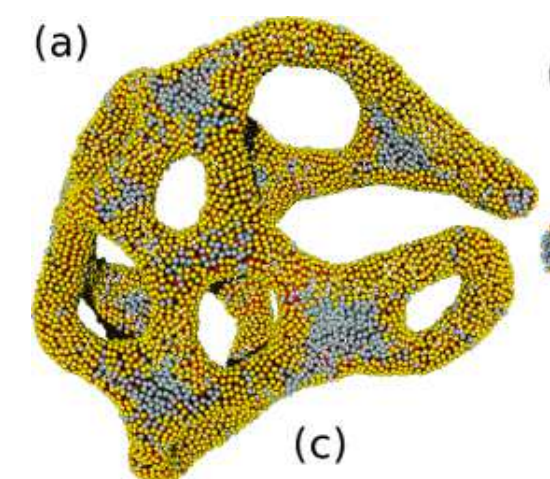

(b)

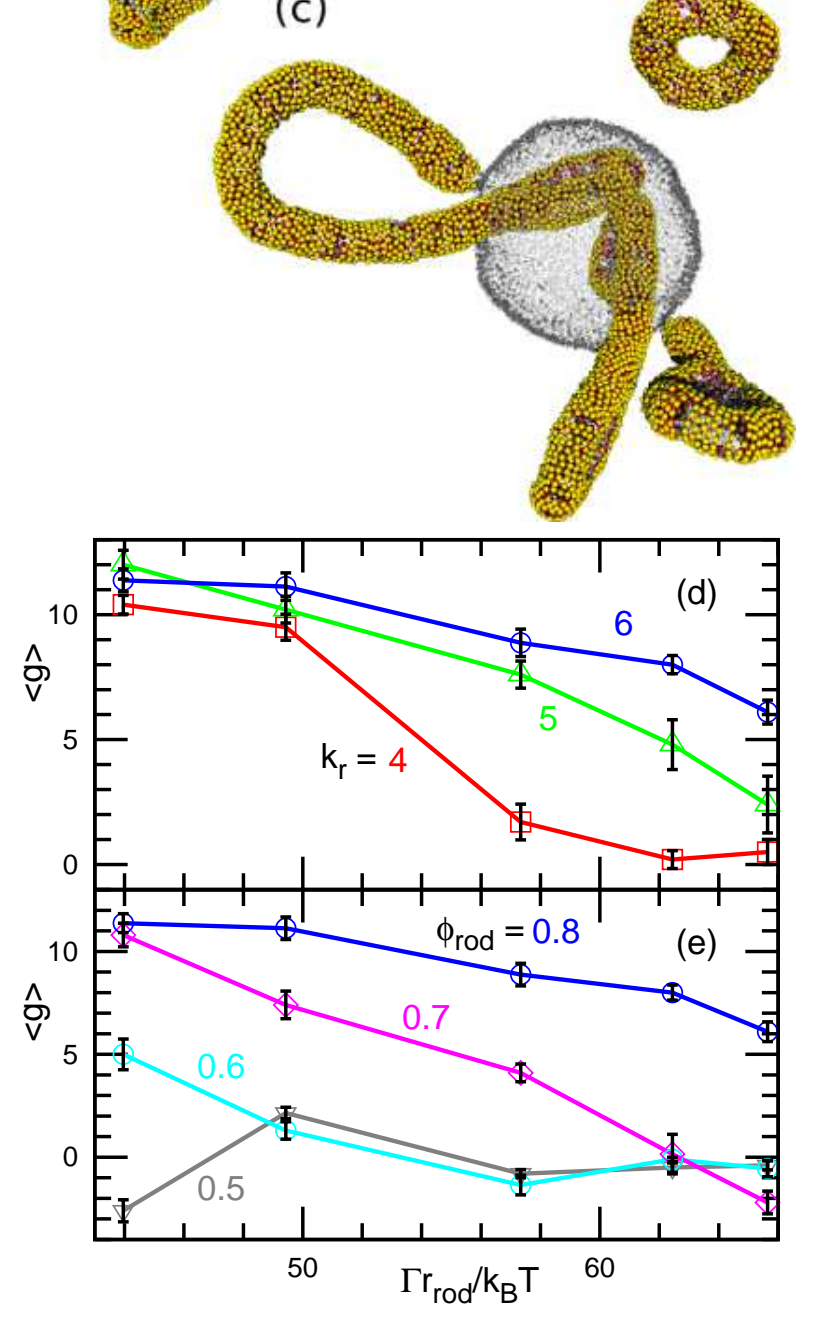

FIG. 8: (Color online) Membrane rupture at $C_{\text {rod }} R_{\text {ves }}=$ -6.2 and $N=9,600$. (a)-(c) Snapshots of vesicles. (a) Genus-11 vesicle at $\Gamma r_{\text {rod }} / k_{\mathrm{B}} T=49, k_{\mathrm{r}}=4$, and $\phi_{\text {rod }}=0.8$. (b) Invaginated genus- 0 vesicle at $\Gamma r_{\text {rod }} / k_{\mathrm{B}} T=62, k_{\mathrm{r}}=4$, and $\phi_{\text {rod }}=0.8$. (c) Ruptured membrane at $\Gamma r_{\text {rod }} / k_{\mathrm{B}} T=49$, $k_{\mathrm{r}}=6$, and $\phi_{\text {rod }}=0.5$. Membrane particles are displayed by nontransparent or transparent gray particles in (a) and (b) or (c), respectively, for clarity. (d)-(e) Mean number of the genus $\langle g\rangle$ of vesicles as functions of the line tension $\Gamma$. (d) $k_{\mathrm{r}}=4,5$, and 6 for $\phi_{\text {rod }}=0.8$. (e) $\phi_{\text {rod }}=0.5,0.6,0.7$, and 0.8 for $k_{\mathrm{r}}=6$.

of proteins onto the membrane is also important for obtaining vesicle rupture in experiments. 


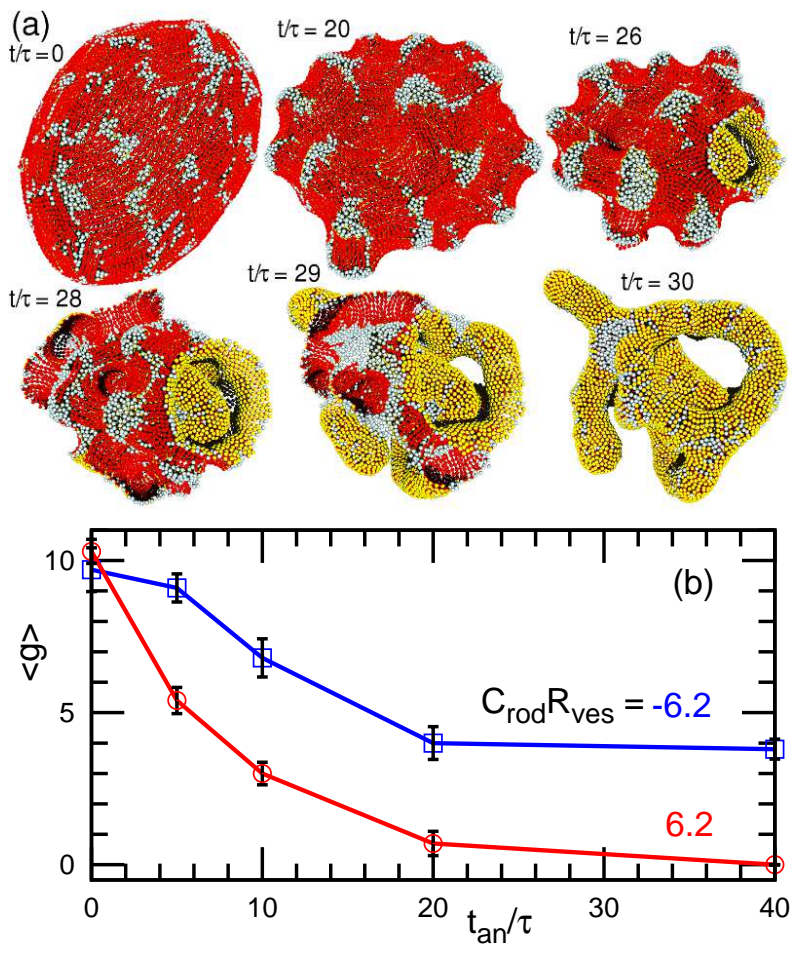

FIG. 9: (Color online) Annealing time, $t_{\mathrm{an}}$, dependence of membrane rupture at $\Gamma r_{\text {rod }} / k_{\mathrm{B}} T=44, k_{\mathrm{r}}=8, \phi_{\text {rod }}=0.8$, and $N=9,600$. (a) Sequential snapshots of a vesicle for $t / \tau=$ $0,20,26,28,29$, and 30 at $C_{\mathrm{rod}} R_{\mathrm{ves}}=-6.2$ and $t_{\mathrm{an}} / \tau=$ 40. Membrane particles are displayed by nontransparent gray particles. (b) Mean number of the genus $\langle g\rangle$ as functions of $t_{\text {an }}$ for $C_{\text {rod }} R_{\text {ves }}=-6.2$ and 6.2 .

We also investigated vesicle rupture by protein rods with a curvature opposite to that of the vesicle curvature (see Figs. 8 and 9). At first, the rods induce many tubular invaginations into the inside of the vesicle [see Fig. 8(b)]. For large $\Gamma$ with relatively small $k_{\mathrm{r}}$, the membrane is not ruptured and densely-packed invaginations remain. Similar invaginations were observed by electron microscopy for liposomes with I-BAR proteins [13]. For small $\Gamma$ or large $k_{\mathrm{r}}$, the membrane is ruptured and subsequently the inside surface of the vesicle turns to the outside [see Fig. 9(a)]. At high $\phi_{\text {rod }}$, this inversion (inside out) occurs completely and a high-genus vesicle is formed [see Fig. 8(a)]. However, at low $\phi_{\text {rod }}$, the inversion is only partial and rod-free membrane regions are not inverted, leading to division and partial connection of the membranes. In the membrane shown in Fig. 8(c), the rod-free membrane forms a pored vesicle and inverted membrane tubes partially remain in the vesicle. The ends of the tubes can be connected to the vesicle, but some of them are eventually pinched off. For genus estimation, complete membrane fission and partial connection are counted as -1 and -0.5 , respectively $[g=-2$ in the case of Fig. 8(c)]. Since the bending energy of an initially spherical vesicle is larger than the positive rod curvature $C_{\text {rod }}$ with the same amplitude, membrane rupture occurs at smaller $\phi_{\text {rod }}, k_{\mathrm{r}}$, or larger $\Gamma$. Even when $C_{\text {rod }}(t)$ is annealed slowly, high-genus vesicles are still formed [see Fig. 9(b)].

\section{SUMMARY}

We have investigated the shape transformation of vesicles and membrane tubes induced by protein rod assembly. As the rod curvature $C_{\text {rod }}$ increases, the protein rods in the membrane tube assemble by two steps; first in the azimuthal direction and next in the longitudinal direction. These assemblies occur at lower $C_{\text {rod }}$ for stiffer rods and/or higher rod density. Compared to the anisotropic rods, laterally isotropic inclusions assemble weakly. The isotropic inclusions assemble only when they induce very large bending rigidity locally; The inclusions with small spontaneous curvatures assemble in flatter regions of an elliptic membrane tube and oblate vesicle, while inclusions with large spontaneous curvatures induce membrane fission into vesicles. The rod-rod excluded-volume interactions make protein rods that are closer than $r_{\text {rod }} / 2$ align in the same orientation. Thus, the elongated shapes of membrane-reshaping proteins also assist protein assembly.

When the protein rods induce a large membrane stress, the membrane can be ruptured and high-genus vesicles form. We have clarified that membrane rupture is induced by the large bending stiffness of the rods, high density, rapid protein adhesion, and/or low line tension of the membrane edge. Thus, the choice of lipids is also important. When $C_{\text {rod }}$ is negative with respect to the initial vesicle curvature, the membrane inversion also results in vesicle division as well as in the formation of high-genus vesicles. Our simulation results suggest that rapid exposure of liposomes to a protein solution is a key factor for observing high-genus liposomes due to protein-induced membrane rupture. Vesicle inversion was previously observed during lysis of a liposome by detergents [67]. Here, we have demonstrated that the protein adhesion can also induce the vesicle inversion.

Here, we considered that the spontaneous (side) curvature and bending rigidity of the rods perpendicular to the rod axis are the same as the other membrane regions. Excluded-volume and other interactions between the proteins or between the protein and membrane can generate effective side spontaneous curvatures. When the rod and side curvatures are in opposite directions, saddle-shaped membranes, such as egg-carton [37, 38], ring, and network structures [50], can be stabilized. Thus, anisotropic inclusions can induce much more variety in membrane structures than isotropic inclusions.

\section{Acknowledgments}

The replica exchange simulations were carried out on SGI Altix ICE 8400EX and ICE XA at ISSP Super- 
computer Center, University of Tokyo. This work was partially supported by a Grant-in-Aid for Scientific Research on Innovative Areas "Fluctuation \& Structure"
(No. 25103010) from the Ministry of Education, Culture, Sports, Science, and Technology of Japan.
[1] H. T. McMahon and J. L. Gallop, Nature 438, 590 (2005).

[2] Y. Shibata, J. Hu, M. M. Kozlov, and T. A. Rapoport, Annu. Rev. Cell Dev. Biol. 25, 329 (2009).

[3] G. Drin and B. Antonny, FEBS Lett. 584, 1840 (2010).

[4] T. Baumgart, B. R. Capraro, C. Zhu, and S. L. Das, Annu. Rev. Phys. Chem. 62, 483 (2011).

[5] L. Johannes, C. Wunder, and P. Bassereau, Cold Spring Harbor Perspect. Biol. 6, a016741 (2014).

[6] H. T. McMahon and E. Boucrot, J. Cell Sci. 128, 1065 (2015).

[7] T. Itoh and P. De Camilli, Biochim. Biophys. Acta 1761, 897 (2006).

[8] M. Masuda and N. Mochizuki, Semin. Cell Dev. Biol. 21, 391 (2010).

[9] H. Zhao, A. Pykäläinen, and P. Lappalainen, Curr. Opin. Cell Biol. 23, 14 (2011).

[10] C. Mim and V. M. Unger, Trends Biochem. Sci. 37, 526 (2012).

[11] M. Simunovic, G. A. Voth, A. Callan-Jones, and P. Bassereau, Trends Cell Biol. 25, 780 (2015).

[12] B. J. Peter, H. M. Kent, I. G. Mills, Y. Vallis, P. J. G. Butler, P. R. Evans, and H. T. McMahon, Science 303, 495 (2004).

[13] P. K. Mattila, A. Pykäläinen, J. Saarikangas, V. O. Paavilainen, H. Vihinen, E. Jokitalo, and P. Lappalainen, J. Cell Biol. 176, 953 (2007).

[14] A. Frost, R. Perera, A. Roux, K. Spasov, O. Destaing, E. H. Egelman, P. De Camilli, and V. M. Unger, Cell 132, 807 (2008).

[15] Q. Wang, M. V. A. S. Navarro, G. Peng, E. Molinelli, S. L. Goh, B. L. Judson, K. R. Rajashankarc, and H. Sondermann, Proc. Natl. Acad. Sci. USA 106, 12700 (2009).

[16] C. Zhu, S. L. Das, and T. Baumgart, Biophys. J. 102, 1837 (2012).

[17] Y. Tanaka-Takiguchi, T. Itoh, K. Tsujita, S. Yamada, M. Yanagisawa, K. Fujiwara, A. Yamamoto, M. Ichikawa, and K. Takiguchi, Langmuir 29, 328 (2013).

[18] Z. Shi and T. Baumbart, Nature Comm. 6, 5974 (2015).

[19] C. Prévost, H. Zhao, J. Manzi, E. Lemichez, P. Lappalainen, A. Callan-Jones, and P. Bassereau, Nature Comm. 6, 8529 (2015).

[20] J. M. Isas, M. R. Ambroso, P. B. Hegde, J. Langen, and R. Langen, Structure 23, 873 (2015).

[21] J. Adam, N. Basnet, and N. Mizuno, Sci. Rep. 5, 15452 (2015).

[22] R. Lipowsky, Faraday Discuss. 161, 305 (2013).

[23] W. T. Góźdź, J. Phys. Chem. B 110, 21981 (2006).

[24] R. Phillips, T. Ursell, P. Wiggins, and P. Sens, Nature 459, 379 (2009).

[25] M. J. Greenall and G. Gompper, Langmuir 27, 3416 (2011).

[26] H. Noguchi, Soft Matter 8, 8926 (2012).

[27] S. Aimon, A. Callan-Jones, A. Berthaud, M. Pinot, G. E. Toombes, and P. Bassereau, Dev. Cell 28, 212 (2014).

[28] B. J. Reynwar, G. Ilya, V. A. Harmandaris, M. M.
Müller, K. Kremer, and M. Deserno, Nature 447, 461 (2007).

[29] T. Auth and G. Gompper, Phys. Rev. E 80, 031901 (2009).

[30] A. Šarić and A. Cacciuto, Phys. Rev. Lett. 108, 118101 (2012).

[31] J. Gómez-Llobregat, F. Elías-Wolff, and M. Lindén, Biophys. J. 110, 197 (2016).

[32] P. B. Canham, J. Theor. Biol. 26, 61 (1970).

[33] W. Helfrich, Z. Naturforsch 28c, 693 (1973).

[34] J.-B. Fournier, Phys. Rev. Lett. 76, 4436 (1996).

[35] D. Kabaso, E. Gongadze, P. Elter, U. van Rienen, J. Gimsa, V. Kralj-Iglič, and A. Iglič, Mini Rev. Med. Chem. 11, 272 (2011).

[36] A. Iglič, H. Hägerstrand, P. Veranič, A. Plemenitaš, and V. Kralj-Iglič, J. Theor. Biol. 240, 368 (2006).

[37] P. G. Dommersnes and J. B. Fournier, Eur. Phys. J. B 12, 9 (1999).

[38] P. G. Dommersnes and J. B. Fournier, Biophys. J. 83, 2898 (2002).

[39] Y. Schweitzer and M. M. Kozlov, PLoS Comput. Biol. 11, e1004054 (2015).

[40] P. D. Blood and G. A. Voth, Proc. Natl. Acad. Sci. USA 103, 15068 (2006).

[41] A. Arkhipov, Y. Yin, and K. Schulten, Biophys. J. 95, 2806 (2008).

[42] G. Khelashvili, D. Harries, and H. Weinstein, Biophys. J. 97, 1626 (2009).

[43] H. Yu and K. Schulten, PLoS Comput. Biol. 9, e1002892 (2013)

[44] M. Simunovic, A. Srivastava, and G. A. Voth, Proc. Natl. Acad. Sci. USA 110, 20396 (2013).

[45] M. Simunovic and G. A. Voth, Nature Comm. 6, 7219 (2015).

[46] N. Ramakrishnan, J. H. Ipsen, and P. B. Sunil Kumar, Soft Matter 8, 3058 (2012).

[47] N. Ramakrishnan, P. B. Sunil Kumar, and J. H. Ipsen, Biophys. J. 104, 1018 (2013).

[48] H. Noguchi, EPL 108, 48001 (2014).

[49] H. Noguchi, J. Chem. Phys. 143, 243109 (2015).

[50] H. Noguchi, Sci. Rep. 6, 20935 (2016).

[51] G. S. Ayton, E. Lyman, V. Krishna, R. D. Swenson, C. Mim, V. M. Unger, and G. A. Voth, Biophys. J. 97, 1616 (2009).

[52] M. Simunovic, C. Mim, T. C. Marlovits, G. Resch, V. M. Unger, and G. A. Voth, Biophys. J. 105, 711 (2013).

[53] H. Noguchi, J. Phys. Soc. Jpn. 78, 041007 (2009).

[54] H. Noguchi and G. Gompper, Phys. Rev. E 73, 021903 (2006).

[55] H. Shiba and H. Noguchi, Phys. Rev. E 84, 031926 (2011).

[56] H. Noguchi, EPL 102, 68001 (2013).

[57] M. P. Allen and D. J. Tildesley, Computer Simulation of Liquids (Clarendon Press, Oxford, 1987).

[58] H. Noguchi, J. Chem. Phys. 134, 055101 (2011).

[59] S. Ramadurai, A. Holt, V. Krasnikov, G. van den Bo- 
gaart, J. A. Killian, and B. Poolman, J. Am. Chem. Soc. 131, 12650 (2009).

[60] K. Hukushima and K. Nemoto, J. Phys. Soc. Jpn. 65, 1604 (1996).

[61] Y. Okamoto, J. Mol. Graph. Model. 22, 425 (2004).

[62] H. Wu, H. Shiba, and H. Noguchi, Soft matter 9, 9907 (2013).

[63] T. V. Tolpekina, W. K. den Otter, and W. J. Briels, J. Chem. Phys. 121, 8014 (2004).
[64] P. Fromherz, Chem. Phys. Lett. 94, 259 (1983).

[65] M. Hu, J. J. Briguglio, and M. Deserno, Biophys. J. 102, 1403 (2012).

[66] K. M. Nakagawa and H. Noguchi, Soft matter 11, 1403 (2015).

[67] F. Nomura, M. Nagata, T. Inaba, H. Hiramatsu, H. Hotani, and K. Takiguchi, Proc. Natl. Acad. Sci. USA 98, 2340 (2001). 